\title{
ANÁLISE DOS EFEITOS DO ATRASO E DA PROBABILIDADE DO REFORÇO EM CONDIÇÕES COM ESQUEMAS CONCORRENTES SIMPLES
}

\section{THE ANALYSIS OF THE EFFECTS OF REINFORCER DELAY AND REINFORCER PROBABILITY IN CONDITIONS WITH SIMPLE CONCURRENT SCHEDULES}

\author{
Daniel Carvalho de Matos \\ Pontifícia Universidade Católica de SÃo PaUlo e Universidade Nove de JULHo \\ NILZA MICHELETTO \\ PONTIFÍCIA UNIVERSIDADE CATÓLICA DE SÃo PAULO
}

\begin{abstract}
RESUMO
O estudo avaliou semelhanças nos efeitos de atraso e probabilidade do reforço sobre escolhas em esquemas concorrentes. Foram programadas para três participantes universitários escolhas em condições com magnitude e atraso do reforço (condição 1) e para outros três em condição com magnitude e probabilidade do reforço (condição 2). Na Fase $\mathrm{CRF} / \mathrm{CRF}$, a alternativa com menor magnitude e menor atraso (ou menor magnitude e maior probabilidade) deveria ser a mais escolhida. Depois vigorou a Fase FR/FR, em que a reversão da escolha foi identificada, exceto para um participante (condição 2), sugerindo semelhanças entre os parâmetros. As demais fases envolveram esquemas CRF, FR com blackout e FI cujos efeitos foram medidos. Os dados revelaram mudança de padrão de escolha para dois participantes (condição 2).

Palavras-chave: Escolha, autocontrole, reversão da escolha, atraso do reforço, probabilidade do reforço.
\end{abstract}

The research assessed similarities between reinforcer delay and probability effects over choices in concurrent schedules. Conditions with reinforcer amount and delay were programmed for three college students (condition 1) and, for other three students, conditions with reinforcer amount and probability (condition 2). In phase CRF/CRF, the component with smaller amount and immediate (or smaller amount and higher probability) should be the most chosen one. Then, a phase FR/FR phase was programmed, in which choice reversals were identified. This suggests similarities between the parameters. The remaining phases involved CRF, FR with blackout and FI schedules and the effects over choices were measured. Data revealed changes in response pattern for two participants (condition 2).

Keywords: Choice, self-control, choice reversal, reinforcer delay, reinforcer probability

Correspondência referente ao presente artigo deve ser enviada ao primeiro autor: E-mail: dcmatos23@hotmail.com 
Em situações de tomada de decisão entre esquemas concorrentes, em que indivíduos devem escolher entre uma alternativa correlacionada com um reforçador de maior magnitude e atrasado vs. outra alternativa com reforçador de menor magnitude e imediato, há usualmente maior escolha da segunda alternativa, o que tem recebido o nome de impulsividade (ver Hanna \& Todorov, 2002). Por outro lado, o controle do responder pela primeira alternativa mencionada tem sido denominado de autocontrole. Em diversas pesquisas de analistas do comportamento com não humanos e humanos, tais situações foram investigadas e variáveis foram manipuladas com o objetivo de aumentar o controle do responder pelo componente com reforçador maior e mais atrasado (ou menos provável), produzindo autocontrole (Green \& Rachlin, 1996; Rachlin, Castrogiovanni, \& Cross, 1987; Rachlin \& Green, 1972; Siegel \& Rachlin, 1995). As manipulações desses pesquisadores, em outras palavras, tinham o objetivo de produzir a escolha da alternativa denominada de autocontrole.

A primeira pesquisa experimental sobre o fenômeno foi desenvolvida por Rachlin e Green (1972) usando um procedimento com esquemas concorrentes encadeados e tendo pombos como sujeitos. Cinco pombos foram sujeitos em uma câmara experimental com dois discos de respostas em uma de suas paredes. Cada esquema era representado por uma cadeia com dois elos. O elo inicial apresentou uma condição de escolha entre dois discos brancos (disco da direita vs. disco da esquerda). Para cada disco estava em vigor um esquema de razão fixa (FR25). Ao emitir a $25^{\mathrm{a}}$ e última bicada em um dos dois discos, o pombo tinha acesso ao segundo elo do encadeamento. Caso essa última bicada fosse feita no disco da direita, após a passagem de $\mathrm{T}$ segundos, entraria em vigor, no segundo elo, uma nova condição de escolha. $\mathrm{O}$ pombo deveria escolher entre um disco verde e um vermelho (cada disco em CRF): o disco verde permitia o acesso a um reforçador maior (definido como $4 \mathrm{~s}$ de acesso a alimento) e mais atrasado (após 4s); o disco vermelho permitia o acesso a um reforçador menor (definido como $2 \mathrm{~s}$ de acesso a alimento) e imediato (zero de atraso). Quando se escolhia o disco vermelho, seguiam-se, após o acesso ao reforçador, mais 6 s a fim de que o tempo total em cada tentativa para ambas as condições fosse aproximadamente o mesmo. Caso contrário, as taxas totais de reforçadores obtidos em cada um dos discos seria diferente. Se, no primeiro elo de escolha entre os discos da direita e da esquerda, a $25^{\mathrm{a}}$ bicada fosse feita no disco B, após a passagem de $\mathrm{T}$ segundos, entraria em vigor $\mathrm{o}$ segundo elo que representaria apenas o disco verde, correlacionado com o reforçador maior e atrasado.

$\mathrm{Na}$ pesquisa de Rachlin e Green (1972), a variável $\mathrm{T}$ (definido como o tempo entre o final do primeiro elo e o início do segundo elo) foi aquela que pareceu modular as reversões nas preferências entre os discos da direita e da esquerda. Diversos valores de T que variaram entre $0,5 \mathrm{~s}$ e $16 \mathrm{~s}$ foram utilizados. Quando $\mathrm{T}$ era curto (p. ex., 0,5s), a preferência dos pombos foi exclusiva pelo disco da direita no primeiro elo, seguido pelo disco vermelho no segundo elo, correlacionado com o reforçador menor e imediato. No entanto, quando $\mathrm{T}$ era longo (p. ex., 16s), os pombos escolhiam mais o disco da esquerda no primeiro elo, seguido pelo acesso ao reforçador maior e atrasado do segundo elo. Isto é, 16s entre os elos cumpriu com seu papel de prolongar o tempo até o acesso aos reforçadores no elo terminal da cadeia. Dessa maneira, ambos os reforçadores se tornaram atrasados e a reversão da preferência foi experimentalmente demonstrada: houve agora a preferência pela escolha do reforçador maior e atrasado (autocontrole). O autocontrole foi demonstrado pela reversão da preferência a partir do momento em que ambos os reforçadores ao final da cadeia se tornaram atrasados. Esse modelo de autocontrole recebeu (por Rachlin \& Green, 1972) o nome de compromisso rigoroso (strict commitment) e envolve a eliminação da possibilidade de controle pela alternativa correlacionada com o reforçador menor e imediato.

O compromisso rigoroso foi ainda investigado em um estudo conduzido com universitários (Rachlin, Castrogiovanni, \& Cross, 1987). Uma importante diferença em relação à pesquisa de Rachlin e Green (1972) com pombos foi a não manipulação do parâmetro de atraso do reforço. Em seu lugar foi manipulada a probabilidade do reforço. O objetivo dos autores com isso era o de avaliar uma possível semelhança desses parâmetros em relação aos seus efeitos sobre a escolha. Se os dados sugerissem alguma semelhança, poder-se-ia sugerir que um reforço atrasado seria como um reforço pouco provável e, por outro lado, um reforço imediato seria como um reforço provável. O delineamento envolveu um procedimento de esquemas concorrentes encadeados com dois elos. Diferentes quantias reais de dinheiro foram usadas como reforçadores, assim como diferentes probabilidades de produção dos reforçadores. A variável crítica que representou a possibilidade de passagem entre os elos da cadeia foi a probabilidade (Q), semelhante à variável tempo (T) entre os elos, da pesquisa de Rachlin e Green.

Para o primeiro elo de escolha (entre dois cartões sobre uma mesa, sendo eles A e B), cada participante recebia 20 fichas (10 vermelhas e 10 azuis). $\mathrm{O}$ número de tentativas, portanto, estava pré-determinado. As vermelhas eram fichas de alta probabilidade e as azuis eram fichas de baixa probabilidade de passagem para o segundo elo. Os participantes poderiam apostar as fichas, uma de cada vez, na sequência que quisessem. Ao colocar cada ficha em A ou $\mathrm{B}$, o experimentador girava uma roleta com 18 números e um ponteiro que permitiria, ao participante, avançar para um segundo elo a depender do número no qual o ponteiro da roleta parasse e da probabilidade da ficha apostada. No caso das fichas vermelhas com alta probabilidade, se o ponteiro parasse em um dos números de 1 a 15 , o participante poderia, então, passar para o segundo elo. No caso das fichas azuis com baixa probabilidade, o participante poderia passar para o segundo elo se o ponteiro parasse em um número que fosse de 1 a 3 .

Se houvesse a passagem para o segundo elo após apostar em A, o participante recebia uma ficha branca, que 
poderia ser apostada em dois outros cartões. Esse momento representava a escolha entre reforçador menor e com alta probabilidade (US\$ 1 , se o ponteiro da roleta parasse em um número de 1 a 17) e reforçador maior com baixa probabilidade (US\$ 4, caso o ponteiro parasse em um número de 1 a 5). No entanto, se houvesse a passagem para o segundo elo após uma aposta ter sido feita em B no primeiro elo, isso significaria o comprometimento com o reforçador maior e menos provável do segundo elo (US\$ 5 , se o ponteiro da roleta parasse em um número que fosse de 1 a 5$)$.

Os resultados de Rachlin et al. (1987) revelaram que os participantes tenderam, no princípio, a apostarem cada uma das fichas vermelhas (alta probabilidade). Essas fichas foram consistentemente apostadas em $\mathrm{A}$ e, quando ocorria a passagem para o segundo elo (nova escolha) que era bastante provável, os participantes recebiam a ficha branca e apostavam no cartão que valia US\$ 1 com alta probabilidade. Por outro lado, os participantes apostaram as fichas azuis (baixa probabilidade) consistentemente em B no primeiro elo. A probabilidade de passagem para o segundo elo era baixa, mas quando ocorria, aumentavam as chances de acesso ao reforçador maior e menos provável (5 dólares).

Rachlin et al. (1987) discutem que as fichas vermelhas tiveram uma função semelhante ao $\mathrm{T}$ (tempo entre os elos) da pesquisa de Rachlin e Green quando $\mathrm{T}$ consistia, por exemplo, em $0,5 \mathrm{~s}$ (tempo curto). Os pombos preferiam A no primeiro elo e preferiam o reforçador menor imediato na segunda condição de escolha. Os participantes da pesquisa de Rachlin et al. apostavam cada ficha vermelha em A. Por serem fichas com alta probabilidade, passavam frequentemente para o segundo elo (nova condição de escolha) e escolhiam consistentemente o reforçador menor e mais provável (US\$ 1).

As fichas azuis eram sistematicamente apostadas em B (primeiro elo) e possibilitavam (quando ocorria a passagem de um elo para o outro) o acesso (compromisso) apenas à alternativa com o reforçador maior e menos provável do segundo elo (US\$ 5). A partir dos resultados da pesquisa, Rachlin et al. concluíram que o atraso e a probabilidade do reforçador são parâmetros funcionalmente semelhantes.

Ainda sobre o compromisso rigoroso (apresentado nas pesquisas de Rachlin \& Green, 1972; Rachlin et al., 1987), entretanto, Rachlin (2000) descreve que as situações da vida dos seres humanos não se caracterizam por possibilidades de compromisso rigoroso em geral, podendo ser quebrado. Para o autor, um caso mais extremo de compromisso rigoroso em humanos, seria, por exemplo, a situação de um alcoolista que deliberadamente decide se internar em uma clínica de reabilitação, estabelecendo o compromisso com o não beber. Outras pesquisas em autocontrole envolveram um delineamento com esquemas concorrentes simples (em que um primeiro elo de compromisso não estava presente), como foi o caso do estudo de Siegel e Rachlin (1995) com pombos. Neste caso, havia apenas a situação de escolha entre uma alternativa com reforço maior e atrasado vs. outra com reforço menor e imediato. Há trabalhos que realizaram uma apresentação de várias pesquisas experimentais que exploraram o modelo em que o elo de compromisso não se faz presente (como as relatadas por Hanna \& Todorov, 2002; Logue, 1995).

A pesquisa de Siegel e Rachlin (1995), portanto, representou uma proposta de investigação de uma condição diferente daquela do modelo de compromisso rigoroso (strict commitment) de Rachlin e Green (1972) e Rachlin et al. (1987). Siegel e Rachlin argumentaram que comportamentos de autocontrole podem ser estabelecidos ao se formarem padrões que dificilmente são quebrados quando emitidos frequentemente. Esquemas de razão fixa, que foram utilizados em parte da pesquisa, revelaram efeitos significativos sobre a escolha do componente de autocontrole. $\mathrm{O}$ fato de essa variável favorecer o estabelecimento de um padrão de autocontrole significativo torna importante uma discussão mais apurada acerca do que ficou conhecido como compromisso leve (soft commitment).

As escolhas chamadas de "compromisso leve" de Siegel e Rachlin (1995) se tratam de um modelo em que o autocontrole ocorre a partir do estabelecimento de padrões de comportamento. É o resultado de se comportar frequentemente de uma determinada maneira. Na vida real dos seres humanos pode-se considerar, por exemplo, o caso de alguém que declara preferir chocolate à salada. Neste caso, comer chocolate em vez de salada envolve uma mera questão de preferência. Entretanto, alguém que declare preferir chocolate, mas come a salada, está se autocontrolando. A partir do momento em que a pessoa passa a comer alimentos saudáveis como salada sistematicamente, tende a formar padrões de comportamento muito fortalecidos (Rachlin, 1994, 1995a, 1995b, 2000; Siegel \& Rachlin, 1995). Essa forma de escolha (compromisso) é leve porque pode ser eventualmente quebrada. A pessoa pode, em algum momento, comer o chocolate. Essa visão estaria mais de acordo com as situações de escolha da vida real dos humanos, dado que os padrões de escolha (chamadas de compromisso) tendem a serem constantemente quebrados. No entanto, a quebra do padrão envolve o custo da interrupção de um padrão fortalecido.

Em todas as fases do experimento de Siegel e Rachlin (1995), cada pombo era colocado em uma câmara que continha dois discos de respostas em uma de suas paredes. Um dos discos estava iluminado de verde e o outro de vermelho. Os discos eram apresentados randomicamente alternados de lado a lado em cada tentativa. Para dois pombos, o disco vermelho estava correlacionado com $2,5 \mathrm{~s}$ de acesso à ração atrasados em $0,5 \mathrm{~s}$ (reforçador menor e imediato), seguido por um blackout de $5 \mathrm{~s}$; o disco verde, por sua vez, estava correlacionado com $4,5 \mathrm{~s}$ de acesso ao alimento atrasados por 3,5s de blackout (reforçador maior e atrasado). Para os outros dois pombos, ocorria o oposto.

$\mathrm{O}$ experimento contou com um procedimento de linha de base, correspondendo a quatro das sete fases da pesquisa (Fases 1, 3, 5 e 7). Nesses casos, a tarefa implicou na escolha de uma das duas alternativas (disco 
verde vs. disco vermelho), utilizando um esquema de CRF para acesso às consequências diferenciais. A segunda fase experimental envolveu a manipulação de esquemas de razão fixa concorrentes (FR31 em cada disco). Neste caso, cada pombo poderia distribuir livremente suas respostas entre os dois discos. Entretanto, o critério seria cumprido em apenas um dos discos, no caso, aquele onde a $31^{\mathrm{a}}$ bicada tivesse sido emitida. Cada sujeito, então, tinha acesso às consequências diferenciais da alternativa onde o critério do FR31 era cumprido. A quarta fase do experimento envolveu o que chamaram de esquemas SigFR31 concorrentes e foi quase idêntica à segunda fase de esquemas FR31 concorrentes. A única diferença de um SigFR31 para um FR31 estava no fato que, no SigFR31, após a $30^{\mathrm{a}}$ bicada em qualquer um dos dois discos, esses discos eram desativados, escurecidos por 1 s e a luz do teto era iluminada por 1s. Após esse breve blackout, a luz do teto era desativada e os discos verde e vermelho, reativados. A próxima bicada do pombo em um dos dois discos permitia o acesso às suas consequências diferenciais. Por fim, a sexta fase do experimento envolveu a programação de esquemas concorrentes de intervalo fixo (FI30s em cada disco). Neste caso, cada um dos dois discos permanecia iluminado por um período de 30s segundos. A primeira resposta de escolha de um dos discos que fosse emitida após os 30 s produzia as consequências diferenciais do disco escolhido. Os autores manipularam FIs concorrentes com intervalos de 30 s porque era, aproximadamente, o tempo que cada pombo levava para cumprir o critério de reforçamento na fase com FR31 concorrentes.

O critério de mudança de uma fase para outra foi o seguinte: após a passagem de pelo menos 15 sessões, nas últimas cinco sessões, cada sujeito deveria ter escolhido uma das alternativas em mais de $50 \%$ das tentativas. Se, depois das 15 sessões, o critério não tivesse sido atingido, continuava-se com a mesma fase em vigor até que cinco sessões sucessivas tivessem ocorrido, sendo que, nesse caso, uma das alternativas deveria ter sido escolhida em mais do que $50 \%$ das tentativas. Se tal critério não tivesse sido alcançado dentro de um total de 40 sessões, assumiase uma indiferença relativa e alterava-se a fase experimental.

A partir da análise dos percentuais de escolha da alternativa associada ao reforçador menor e imediato em cada fase pela qual passou cada sujeito, Siegel e Rachlin (1995) observaram que a fase com esquemas FR31 concorrentes foi a que resultou em maior percentual de seleção do reforçador maior e atrasado $(64 \%$ das tentativas), significando maior autocontrole. Na fase de esquemas SigFR31 concorrentes, cerca de metade das tentativas envolveu a seleção do reforçador maior e atrasado (autocontrole). A fase com esquemas concorrentes FI30 resultou num percentual menos expressivo de seleção do reforçador maior e atrasado (25\% das tentativas). As fases de CRF concorrentes revelaram uma preferência ainda maior pelo reforçador menor imediato em $95 \%$ das tentativas de escolha.

Portanto, a manipulação dos esquemas de razão fixa FR31 concorrentes foi a variável crítica para o estabelecimento do autocontrole nesse estudo. A razão de Siegel e Rachlin (1995) terem manipulado os esquemas FR31 foi a seguinte: esquemas de razão fixa alta usualmente resultam em padrões de comportamento muito rígidos, fortalecidos. Depois do reforço, há normalmente uma pausa (cuja duração é proporcional ao número de respostas necessárias para o cumprimento do critério de reforçamento). Depois, há um responder rápido que se estende até que o critério seja atingido (Ferster \& Skinner, 1957; Rachlin, 2000). O acesso ao reforçador através dos esquemas FR31 concorrentes se tornava atrasado nos dois discos por conta do tempo que os pombos precisavam para o cumprimento do critério. A variável magnitude do reforçador se tornava assim um parâmetro mais crítico para as escolhas, daí o maior percentual de seleção do reforçador maior atrasado. Além disso, os esquemas de reforçamento concorrentes de razão fixa alta tendem a gerar forte preferência por uma das alternativas de escolha, implicando em grande resistência à mudança. Os pombos não mudavam de disco após terem iniciado o FR. Esse argumento justifica o estabelecimento de padrões comportamentais rígidos cuja mudança é custosa (Ferster \& Skinner, 1957; Green \& Rachlin, 1996; Rachlin, 1994, 1995a, 1995b, 2000; Siegel \& Rachlin, 1995).

A fase referente ao esquema SigFR31 e FI30 segundos concorrentes foram delineadas com o objetivo de produzir um relaxamento no padrão rígido estabelecido pelo FR31. A partir do resultado do SigFR31 com um breve blackout de $1 \mathrm{~s}$ antes de o critério poder ter sido cumprido, observou-se que, embora as escolhas do reforçador maior e atrasado tenham diminuído, o padrão foi mantido na medida em que cerca de metade das escolhas envolveu o controle pelo reforçador maior e atrasado. $\mathrm{Na}$ fase com esquemas FI 30 segundos concorrentes, o padrão foi visivelmente alterado, dado que o maior percentual de escolhas passou a ser da alternativa associada ao reforçador menor e imediato. Um esquema FI tende a gerar breves pausas no responder, seguidas por longos jorros de responder rápido (Ferster \& Skinner, 1957; Rachlin, 2000). Como, nesse esquema, houve várias interrupções em função das pausas, os pombos de Siegel e Rachlin (1995) tinham muitas oportunidades para alternarem o responder entre os dois discos.

Embora a pesquisa de Siegel e Rachlin (1995) tenha avaliado os efeitos da manipulação de esquemas de razão fixa concorrentes como geradores de padrões de comportamentos de autocontrole em pombos, tal modelo não foi, até então, aplicado em participantes humanos. Uma análise sobre isso se justificaria para avaliar se os dados com humanos replicariam aqueles produzidos com pombos. Além disso, a mesma tarefa poderia ser também aplicada em humanos, trocando-se o atraso pela probabilidade do reforçador com o intuito de avaliar se haveria uma relação entre os efeitos de cada parâmetro (atraso e probabilidade) sobre a escolha.

Diante do exposto, a presente pesquisa avaliou o estabelecimento de relações de autocontrole com base no compromisso leve e envolveu a escolha entre dois componentes (reforçador maior e atrasado ou pouco provável vs. reforçador menor e imediato ou muito 
provável). Foram manipulados esquemas de reforçamento semelhantes aos que foram utilizados na pesquisa de Siegel e Rachlin (1995) com pombos (CRF, FR, SigFR e FI), mas, para os presentes estudos, os participantes foram humanos. O objetivo foi de (1) avaliar se, após maior escolha em esquema concorrente de reforçamento continuo (CRF/CRF), por reforço imediato e menor ou menor e muito provável do que por reforço maior e maior ou atrasado e pouco provável, a manipulação de esquemas de reforçamento intermitente concorrentes de razão fixa FR/FR concorrentes resultaria em reversão de preferência para maior escolha de reforço maior e mais atrasado ou maior e pouco provável e também (2) avaliar se a manipulação de outros esquemas de reforçamento concorrentes (SigFR, FI) resultaria em mudanças no padrão de respostas estabelecido pelo FR.

\section{MÉTODO}

\section{Participantes}

Participaram seis adultos universitários de uma universidade particular na cidade de São Paulo. Os participantes assinaram um termo de consentimento para a realização da pesquisa.

\section{Material e Local}

Um software de computador foi desenvolvido para a realização da pesquisa e foi utilizado em um computador portátil com monitor e mouse. A primeira tela envolvia dois botões (azul e amarelo). Após respostas de clicar em um dos botões, se seguia uma tela preta e, após $\mathrm{N}$ segundos (para a condição em que foram manipulados atraso e magnitude do reforçador) ou imediatamente (para condição e que foram manipulados probabilidade e magnitude do reforçador), era liberada uma animação, consistindo em três figuras de frutas ou outras figuras como moedas apresentadas no centro da tela, girando por um período de $2 \mathrm{~s}$, que eram finalizados com um som de moedas e o aparecimento de três figuras iguais na tela. A animação era semelhante aos sorteios que são característicos de jogos de azar como o caça níqueis. Pontos eram adicionados a um registrador na parte inferior da tela. Na Figura 1, de forma esquemática estão representadas as sequência de telas apresentadas em uma tentativa.

As sessões experimentais eram realizadas em uma sala, que contava com uma mesa na qual estava um computador e duas cadeiras em frente à mesa, uma para o participante e outra para o experimentador.

\section{Procedimento}

Duas condições foram investigadas: uma que envolveu a manipulação dos parâmetros atraso e magnitude do reforçador; outra que envolveu os parâmetros de probabilidade e magnitude. Para três participantes, o atraso do reforço foi manipulado ao longo das condições. Para os outros três participantes, a probabilidade do reforço foi manipulada ao invés do atraso.

Para todas as fases poderiam ocorrer até quatro blocos de 16 tentativas. As quatro primeiras tentativas do primeiro bloco eram sempre forçadas para os participantes conhecerem as consequências diferenciais de cada alternativa dos componentes do esquema concorrente e as outras eram livres. Nas tentativas forçadas, quando a tela com dois componentes de escolha (quadrados azul e amarelo) era apresentada, em duas das tentativas, apenas o responder em um dos componentes produzia consequência. Respostas no outro componente não produziam qualquer consequência. Nas duas tentativas seguintes acontecia uma inversão. Nas tentativas de escolha livre (14 restantes) era possível operar sobre ambos os componentes e acessar as consequências diferenciais associadas a cada um.

$\mathrm{O}$ experimento era realizado em várias sessões. $\mathrm{O}$ experimentador permanecia na sala ao longo de cada sessão. As instruções apresentadas para cada participante no início da pesquisa foram as seguintes:

"Você vai realizar uma tarefa de jogo no computador, envolvendo cliques entre dois quadrados. Os cliques produzirão uma breve animação e pontos serão adicionados a um contador. Esses pontos serão trocados por dinheiro ao final de uma sessão. Cada ponto valerá 1 centavo."

Os participantes foram expostos a nove fases. Todas elas envolveram esquemas concorrentes:

Fase 1: Magnitude

A tela inicial envolveu a escolha entre um quadrado azul correlacionado com um reforçador de menor magnitude (5 pontos) e um quadrado amarelo associado a um reforçador maior (10 pontos). Blocos de 16 tentativas eram apresentados aos participantes e o critério de aprendizagem estabelecido consistia em $68,75 \%$ das respostas alocadas no componente amarelo com o reforçador de maior magnitude. Caso o critério não fosse obtido após a realização de quatro blocos, o participante era dispensado da pesquisa.

Fase 2: Atraso ou Probabilidade

A tela inicial envolveu a escolha entre um quadrado azul versus outro amarelo. As magnitudes associadas a azul e amarelo eram iguais (5 pontos), mas, nos casos em que o atraso do reforçador era manipulado, a diferença estava nos atrasos. Não houve atraso para o acesso ao reforçador associado ao quadrado azul, mas houve $15 \mathrm{~s}$ de espera após o acesso ao reforçador. No caso do quadrado amarelo, o atraso para o acesso ao reforçador era de $15 \mathrm{~s}$. A fase era encerrada com pelo menos $68,75 \%$ das respostas no componente azul. Se após quatro blocos de tentativas em que esse critério não fosse verificado, o participante era dispensado. Para os participantes para os quais a probabilidade do reforçador era manipulada, a probabilidade de acesso ao reforçador era de $95 \%$ para respostas no quadrado azul e $28 \%$ para respostas no quadrado amarelo. $\mathrm{O}$ critério de encerramento e dispensa do participante era semelhante aos dos participantes expostos à condição atraso do reforçador.

Fase 3: Esquemas CRF (CRF/CRF) concorrentes com magnitude e atraso ou probabilidade do reforço.

Nesta fase estava em vigor, em cada quadrado (azul e amarelo), um esquema de reforçamento contínuo (CRF / CRF) com a manipulação de reforçadores com diferentes magnitudes e atrasos ou probabilidades (a depender da condição a que o participante foi exposto). 


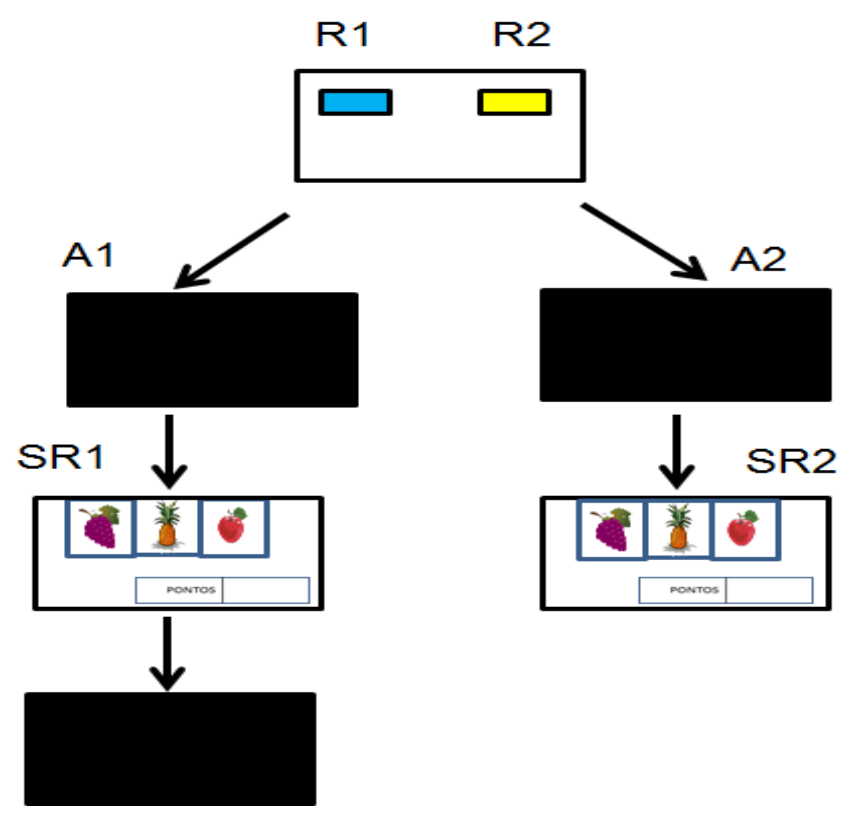

Figura 1. Esquema da sequência de telas apresentadas em uma tentativa na condição atraso. Na condição em que foi manipulada a probabilidade do reforçador, a última tela preta não era apresentada.

$\mathrm{Na}$ condição atraso do reforço, clicar no quadrado azul permitia o acesso ao reforçador menor (5 pontos) e sem atraso (mas com 15 segundos de espera após o acesso ao reforçador) e, clicar no quadrado amarelo, permitia o acesso ao reforçador maior (10 pontos) e mais atrasado (15 segundos). Pelo menos $68,75 \%$ das respostas de escolha dos participantes expostos a essa condição tinham que ser feitas no componente azul com reforço menor e imediato. Para um dos participantes (P3), o atraso do reforço (após R2) ou tempo de espera pós-reforço (após R1) foi de 30 segundos porque 15 segundos foram insuficientes para $\mathrm{O}$ cumprimento do critério na fase. Em função disso, todas as demais fases envolveram esse tempo para esse participante.

Para os participantes expostos à condição probabilidade do reforço, a probabilidade de acesso ao reforçador era de $95 \%$ quando associada ao quadrado azul e $28 \%$ quando associada ao quadrado amarelo. O critério de encerramento era semelhante, com pelo menos $68,75 \%$ das respostas no componente azul.

Conforme pode ser visto na Figura 1, a tela inicial exibia dois quadrados (azul R1 e amarelo R2). Quando o atraso do reforço era manipulado, respostas sobre o quadrado azul (R1) produziam, após 2s de tela escura (A1), a animação por 2 s e 5 pontos - reforçador menor e imediato (SR1). Após isso, havia outra tela preta com duração de 15s. Respostas sobre o quadrado amarelo (R2) produziam, após 17s (A2), a animação, com duração de $2 \mathrm{~s}$, e 10 pontos - reforçador maior e mais atrasado (SR2). A tela de atraso A2 durava 17s (2 a mais) para que o tempo total de cada alternativa do segundo elo (para SR1 e SR2) fosse o mesmo. Ao final de cada sessão, os pontos eram trocados por dinheiro.
Quando a probabilidade do reforço era manipulada, o acesso ao reforçador por meio de cada componente (azul e amarelo) ocorria mediante diferentes probabilidades, sendo $95 \%$ e $28 \%$, respectivamente. As telas pretas (representando A1 e A2) de transição para a animação e acesso ao reforço tinham sempre uma duração de $2 \mathrm{~s}$. Vale destacar que a última tela preta, que permanecia em vigor por 15 segundos na condição em que o atraso do reforço era manipulado, não era apresentada.

Para todos os participantes, os valores manipulados de magnitude, atraso e probabilidade do reforço desta fase vigoraram nas fases subsequentes do experimento.

Para ambas as condições - atraso e probabilidade do reforço, se o critério de encerramento não fosse verificado em até quatro blocos de tentativas, o participante era dispensado da pesquisa. Depois dessa fase, nenhum participante era dispensado quando o critério de cada fase não era alcançado, uma vez que se buscava avaliar os efeitos dos esquemas manipulados sobre as escolhas.

Fase 4: Esquemas FR (FR/FR) concorrentes com magnitude e atraso ou probabilidade do reforço

Nesta fase, foi avaliada se a mudança do esquema de reforçamento de contínuo (CRF) para o esquema de reforçamento intermitente de razão fixa produzia reversão da preferência para maior escolha do componente amarelo com reforço maior atrasado ou pouco provável, a depender da condição. Entrava em vigor um par de esquemas concorrentes em que respostas em ambos os componentes eram reforçadas em esquema de razão fixa (FR10/FR10). A tela inicial envolvia a escolha entre um quadrado azul versus outro amarelo.

Quando o atraso do reforço era manipulado, clicar no quadrado azul produzia em FR o acesso ao 
reforçador menor e sem atraso e, clicar no quadrado amarelo, permitia o acesso ao reforçador maior e mais atrasado. $\mathrm{O}$ encerramento da fase ocorria com a escolha do componente amarelo com o reforçador maior atrasado em pelo menos $68,75 \%$ das tentativas ou após o término dos quatro blocos de tentativas sem que o critério fosse verificado.

Quando a probabilidade do reforço era manipulada, a diferença estava na substituição dos atrasos por probabilidades de acesso a reforço e o critério de encerramento era semelhante. Para ambos os casos (com atraso ou probabilidade do reforço), a razão do FR de cada componente aumentaria de 10 em 10 respostas (até FR80/FR80 se necessário), caso o critério de encerramento não fosse atingido com razão de 10 respostas.

Fase 5: Esquemas CRF (CRF/CRF) concorrentes com magnitude e atraso ou magnitude e probabilidade do reforço.

Idêntica à Fase 3.

Fase 6: Esquemas SigFR (SigFR/SigFR) concorrentes com magnitude e atraso ou magnitude e probabilidade do reforço.

Essa fase contou com as mesmas características da Fase 4, com um par de esquemas concorrentes (SigFR/SigFR). A diferença de um esquema SigFR para um FR era que, após a penúltima resposta de clicar sobre um dos quadrados azul ou amarelo (no SigFR), eles eram desativados por 1s (como em Siegel \& Rachlin, 1995), a tela ficava escura durante esse período e os cliques não tinham quaisquer efeitos. Após esse curto intervalo, a próxima resposta permitia o acesso ao reforçador. A fase era encerrada quando pelo menos $68,75 \%$ das respostas eram alocadas no componente azul com reforçador menor imediato ou menos provável.

Fase 7: Esquemas CRF (CRF/CRF) concorrentes com magnitude e atraso ou probabilidade do reforço.

Idêntica às Fases 3 e 5.

Fase 8: esquema FI (FI / FI) concorrentes com magnitude e atraso ou probabilidade do reforço.

Nesta fase estava em vigor um par de esquemas de intervalo fixo (FI) concorrentes. O intervalo era calculado a partir do tempo que cada participante precisou para cumprir com o critério de razão fixa (FR) do par concorrente da Fase 4 ao qual foi submetido. Os cálculos eram feitos a partir da soma do tempo, que cada participante levava em cada tentativa do último bloco em que a Fase 4 foi encerrada, dividido pelo número total de tentativas. Os intervalos, portanto, podiam variar entre os participantes. Quando a tarefa com o par FI/FI concorrentes estava em vigor, o critério era verificado com pelo menos $68,75 \%$ das respostas no componente azul com reforço menor imediato ou mais provável.

Fase 9: Esquemas CRF (CRF/CRF) concorrentes com magnitude e atraso do reforço.

Idêntica às Fases 3, 5 e 7.

\section{RESULTADOS}

As análises dos dados obedeceram a seguinte sequência de apresentação: (1) os três participantes para os quais magnitude e atraso do reforço foram manipulados e (2) os três participantes para os quais magnitude e probabilidade do reforço foram manipulados.

Condição 1 - magnitude e atraso do reforço

A Figura 2 apresenta os percentuais de escolha das alternativas com o reforçador $(\mathrm{SR}+)$ maior atrasado ao longo de cada uma das fases para os participantes (P1, P2 e P3) expostos à condição magnitude e atraso.

O P1 precisou de três blocos para atingir o critério na fase Magnitude, enquanto P2 e P3 precisaram de apenas um bloco. $\mathrm{Na}$ fase Atraso, P1 atingiu o critério no terceiro bloco de tentativas, enquanto tanto P2 como P3 finalizaram no segundo bloco. Na última fase eliminatória, CRF1, P1 precisou de apenas um bloco para cumprir com critério de escolha de reforço menor imediato, enquanto P2 precisou de três blocos. P3 atingiu o critério no primeiro bloco quando o atraso ou tempo pós-reforço foi de 30 segundos.

A Figura 2 ainda revela, na fase seguinte com esquemas FR concorrentes em que a reversão da preferência foi avaliada, que apenas a razão de 10 respostas (FR10) foi o bastante para que todos os participantes cumprissem com o critério de encerramento da fase. P1, P2 e P3 precisaram de, respectivamente, três, dois e quatro blocos de tentativas.

$\mathrm{Na}$ fase seguinte, com retorno aos esquemas CRF concorrentes (CRF2), não houve, para nenhum participante, um retorno do responder com a maioria das respostas alocadas no componente R1 com reforço menor imediato. Aconteceu justamente o contrário: o padrão de escolhas do componente R2 com reforço maior atrasado, que foi estabelecido na fase com FR, tanto não se modificou como se tornou mais acentuado, diferente do que foi obtido no estudo com animais (Siegel \& Rachlin, 1995). A Figura 2 mostra que em todas as demais fases (SigFR e FI e todas CRF após FR) da condição magnitude e atraso, em que se esperaria uma alteração do padrão estabelecido pelo FR retornando ao responder sob controle do componente $\mathrm{R} 1$, o padrão estabelecido pelo $\mathrm{FR}$ (de escolha da alternativa R2 com reforço maior atrasado) prevaleceu na maior parte dos blocos de tentativas das fases de CRF2, 3 e 4.

Grupo 2 - magnitude e probabilidade do reforço

A Figura 3 apresenta os percentuais de escolha da alternativa com o reforçador de maior magnitude (Fase 1), de menor probabilidade (Fase 2), de menor probabilidade e maior magnitude (fases seguintes) para os participantes P4, P5 e P6.

Conforme pode ser visto na Figura 3, nas três primeiras fases de seleção dos participantes, todos os participantes atingiram o critério de encerramento na fase Magnitude, alocando a maior parte das respostas no componente R2 com reforço de maior magnitude, sendo P4 e P6 no segundo bloco de tentativas e P5 no quarto bloco. Dois outros blocos foram realizados com $\mathrm{P} 4$ por problemas técnicos do software de coleta de dados na alteração das contingências. 


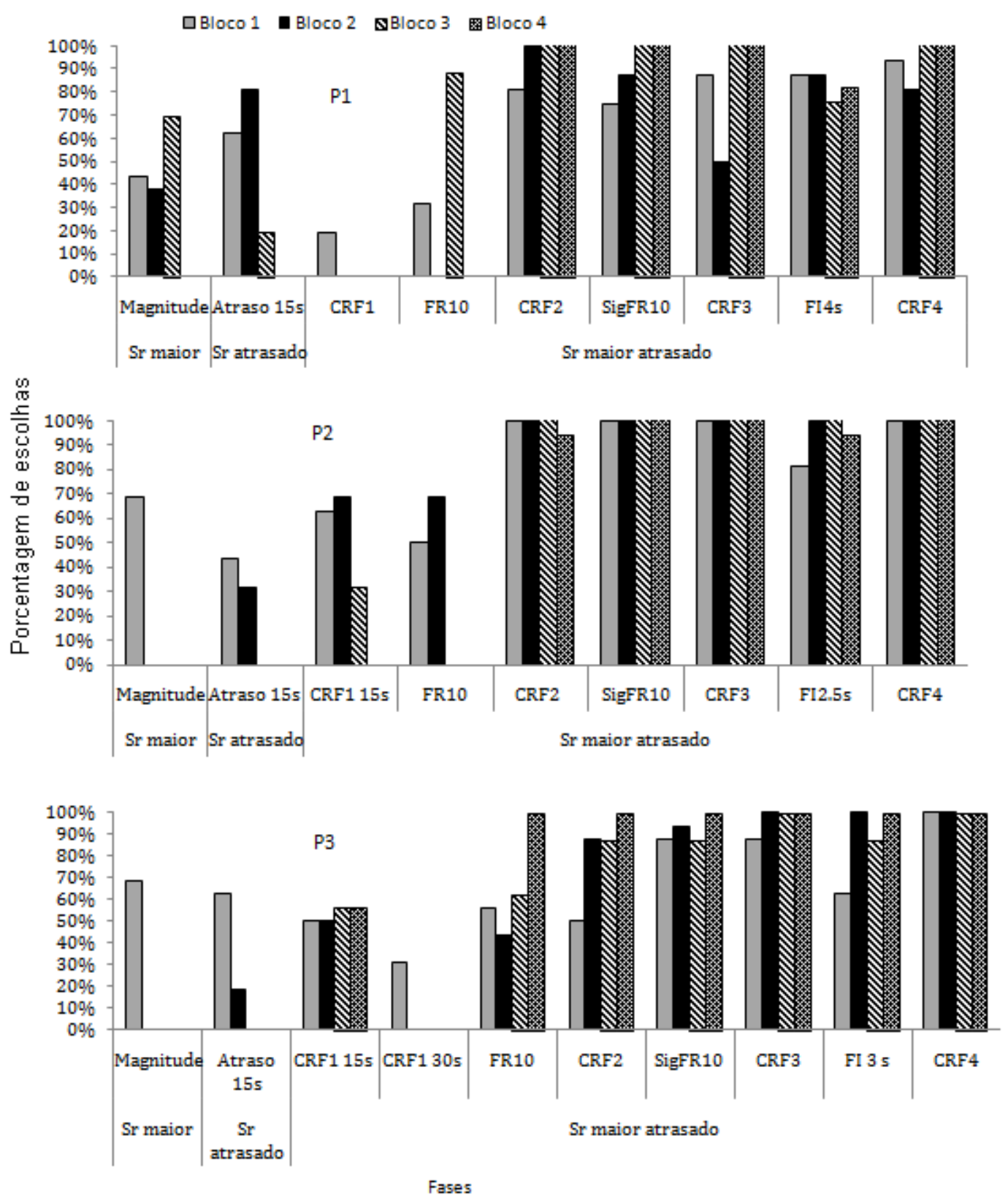

Figura 2. Percentagem de escolhas de reforçador de maior magnitude (Fase 1), maior atraso (Fase 2) e maior atraso e magnitude (fases seguintes) (componente R2), dos participantes P1, P2 e P3, nas fases.

$\mathrm{Na}$ fase seguinte (Probabilidade), todos atingiram o critério, alocando a maior parte das respostas em R1 com reforço mais provável, sendo P4 e P5 no segundo bloco e P6 no primeiro. Na terceira e última fase eliminatória, CRF 1 (com diferenças nas magnitudes e probabilidades dos dois componentes de escolha), P4, P5 atingiram o critério de encerramento da fase no terceiro bloco e P6, no primeiro bloco.

$\mathrm{Na}$ fase seguinte (FR) pode-se observar, de acordo com a Figura 3, que P5 e P6 atingiram o critério, alocando a maior parte das respostas em R2 com reforço maior e menos provável em algum momento da fase de FR / FR concorrentes. P5 conseguiu atingir o critério com um bloco de FR30 / FR30 concorrentes, distribuindo a maior parte das respostas no componente R2. P6 atingiu o critério de encerramento bem mais rapidamente, precisando de apenas um bloco na fase com FR10 / FR10 concorrentes. Por outro lado, no caso do participante P4, verifica-se que o mesmo não atingiu o critério na fase com FR / FR em nenhum momento, passando por todas as razões programadas para o FR (de 10 a 80). Portanto, para esse participante, a reversão da preferência não foi verificada, dado que o mesmo alocou a maior parte de suas respostas no componente R1 com reforço menor e mais provável. 


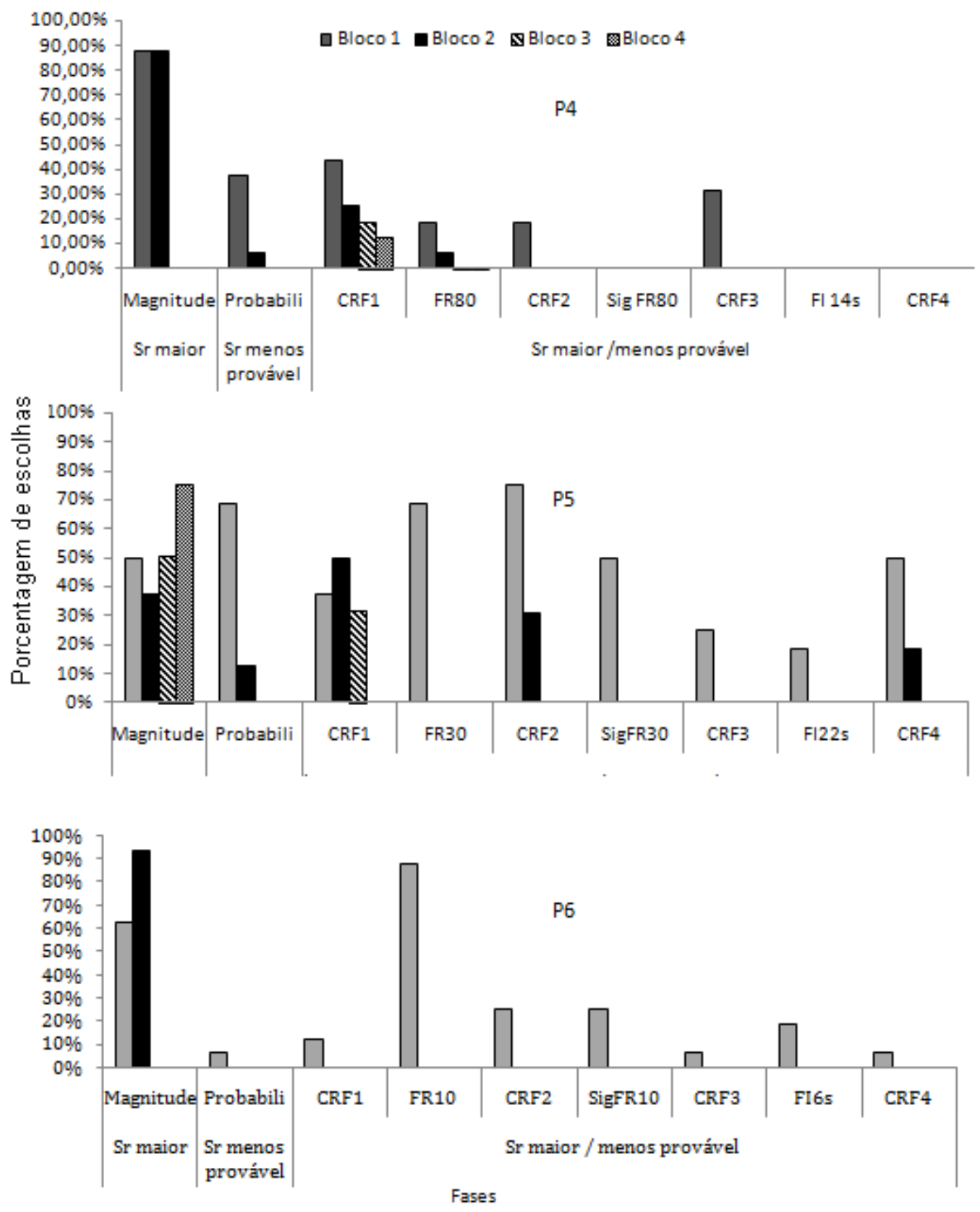

Figura 3. Porcentagem de escolhas do reforço de maior magnitude (Fase 1), menor probabilidade (Fase 2) e maior magnitude e menor probabilidade (fases seguintes) (componente R2) dos participantes P4, P5 e P6, nas fases experimentais. Estão representados os dados do último valor de razão e de intervalo que os participantes realizaram.

Na próxima fase (CRF2) e também nas demais (SigFR, CRF3, FI e CRF4), que se seguiram ao FR/FR concorrentes, foi possível observar que, para P5 e P6, o padrão de respostas mudou, com maior escolha de reforço menor e mais provável. Nesse sentido, esses dados se assemelham aos de condições semelhantes do estudo de Siegel e Rachlin (1995) com pombos, ainda que, neste caso, o atraso do reforço tenha sido manipulado no lugar da probabilidade. P6 não precisou mais do que um bloco de tentativas para cumprir com o critério de encerramento de todas as condições que se seguiram à fase com FR / FR concorrentes. P5, por sua vez, precisou de dois blocos apenas nas condições CRF2 / CRF2 e CRF4 / CFR4. É interessante notar ainda que, no caso de P5, o critério de reversão de preferência para maior escolha de R2 com reforço maior e menos provável nas condições com esquemas FR, apenas aconteceu na fase com esquema FR30, ao contrário do que se verificou no caso de P6 e também de todos os participantes da condição 1 (com atraso do reforço), em que FR10 foi o bastante para o cumprimento do critério de reversão da preferência. No caso de $\mathrm{P} 4$, no entanto, ainda que o padrão de escolhas nas 
condições após aquela com FR / FR concorrentes tenha sido semelhante ao dos outros dois participantes, com maior escolha de reforço menor e mais provável, reitera-se que, para $\mathrm{P} 4$, a fase com $\mathrm{FR}$ não resultou em maior escolha de reforço maior e menos provável e o padrão de escolhas de R1 manteve-se inalterado até a última fase do estudo.

\section{DISCUSSÃO}

No estudo de Siegel e Rachlin (1995) com pombos, a manipulação de esquemas FR31 / FR31 concorrentes foi crítica para o estabelecimento de maior escolha de uma alternativa com reforço maior atrasado. Esses esquemas tendiam a gerar a preferência por essa alternativa, formando um padrão comportamental que, embora pudesse ser alterado, dificilmente isso acontecia. Além disso, como o cumprimento da razão demandava tempo, o FR31, segundo os autores, teria funcionado de forma análoga ao tempo T longo entre os elos da pesquisa de Rachlin e Green (1972), tornando ambos os reforçadores dos esquemas mais atrasados e aumentando o controle do responder pela alternativa com reforço com maior magnitude e maior atraso. O padrão de respostas, entretanto, foi modificado com a introdução de outros esquemas de reforçamento (CRF, SigFR e FI) e o resultado foi a maior escolha da alternativa com reforço menor imediato.

A presente pesquisa representou a proposta de replicar a pesquisa de Siegel e Rachlin (1995) com participantes humanos universitários. Parâmetros e condições semelhantes foram manipulados para esse fim. Para os participantes da condição 1 com magnitude e atraso do reforço, a fase com esquemas FR10/FR10 concorrentes indicou reversão de preferência com maior escolha de reforço maior atrasado de forma semelhante ao que se observou com os pombos de Siegel e Rachlin.

Nas condições seguintes (SigFR, FI e CRF), por outro lado, o padrão de respostas na fase com FR não foi alterado, diferentemente do que se observou no estudo com pombos. Esses resultados podem refletir diferenças no padrão de responder de organismos de diferentes espécies (humanos e pombos). Futuras coletas poderiam ser feitas com algumas manipulações nas condições com esquemas SigFR / SigFR e FI / FI concorrentes. Em primeiro lugar, na fase com SigFR / SigFR, o tempo de blackout na tela (antes de o critério de razão ser cumprido em uma das alternativas) poderia ser superior a 1 segundo, para avaliar se tal alteração implicaria em alguma mudança no padrão de respostas nessa fase. Ou, ainda, o tempo de blackout poderia ser gradualmente aumentado para avaliar um possível momento em que o padrão de respostas mudaria para cada futuro participante. $\mathrm{Na}$ fase com FI / FI concorrentes, intervalos mais longos poderiam ser manipulados para verificar se possíveis mudanças aconteceriam no padrão das respostas. Esses intervalos poderiam ser, por exemplo, gradualmente aumentados.

É importante destacar que o estudo de Matos, Del Poço, Micheletto, Almeida, Rienzo e Panetta (2014), semelhante ao que foi feito com os participantes da condição 1 , mas que manipulou esquemas de reforçamento variáveis ao invés de esquemas fixos, resultou em dados semelhantes. No estudo de Matos et al. (2014), VR10 foi manipulado ao invés de FR10; SigVR10 foi manipulado ao invés de SigFR10; e VI foi manipulado ao invés de FI. Os resultados foram semelhantes, no sentido de que, após a seleção de três participantes em uma fase com esquemas CRF / CRF concorrentes, em que o reforçador menor e imediato foi mais escolhido em relação ao reforçador maior atrasado, a fase com esquemas VR10 / VR10 concorrentes foi programada para avaliar reversão de escolha e isso foi verificado, dado que houve maior escolha da alternativa com reforçador maior atrasado. Em todas as demais condições, que se seguiram àquela com VR10 (CRF, SigVR10 e VI) e que tiveram o objetivo de produzir um padrão de respostas diferente do que foi estabelecido pelo VR 10, o padrão não foi alterado e, mesmo nessas condições e de forma semelhante ao que se observou com P1, P2 e P3 do presente trabalho, houve maior escolha do reforçador maior e atrasado, sugerindo que a manipulação de esquemas de reforço intermitentes fixos e variáveis apresentam efeitos semelhantes com universitários.

Com os participantes da condição 2 (P4, P5 e P6), com os quais a probabilidade do reforço foi manipulada ao invés do atraso, dois (P5 e P6) de três participantes escolheram mais a alternativa com reforçador maior e menos provável na fase com FR / FR concorrentes, de forma semelhante ao que se observou com P1, P2 e P3 com atraso de reforço e com os pombos da pesquisa de Siegel e Rachlin (1995) em que a alternativa com reforço maior e mais atrasado foi a mais escolhida. Esses dados sugerem semelhanças entre os parâmetros de atraso e probabilidade do reforço com relação aos seus efeitos sobre as escolhas em esquemas concorrentes com reforçamento em razão. Não foi possível, entretanto, estabelecer relações precisas entre os dados de humanos e pombos, considerando que, no estudo de Siegel e Rachlin, a probabilidade do reforço não foi manipulada.

Ainda sobre a comparação dos efeitos da probabilidade e do atraso do reforço sobre as escolhas de universitários na literatura, outra pesquisa conduzida por Rachlin et al. (1987) com universitários e com manipulação de probabilidade do reforço em esquemas CRF / CRF concorrentes resultou em dados que sugerem semelhanças entre os parâmetros de atraso e probabilidade do reforço segundo os autores, mas eles sugeriram essas semelhanças com base em comparações dos dados dos universitários da pesquisa com dados de pombos de outra pesquisa (Rachlin \& Green, 1972) em que o atraso do reforço foi manipulado. Além disso, Rachlin et al. não manipularam outros esquemas de reforço como o FR.

Outras pesquisas investigaram os efeitos de manipulação da variável probabilidade do reforço sobre as escolhas de adultos universitários, assim como sua possível relação com o atraso do reforço (Rachlin, Logue, Gibbon, \& Frankel, 1986; Rachlin, Raineri, \& Cross, 1991). Tais pesquisas foram influenciadas pelo modelo de um estudo cognitivista, que avaliou o comportamento de escolha entre recompensas / reforçadores probabilísticas hipotéticas, desenvolvido por Kahneman e Tversky 
(1984). Nesta pesquisa, adultos poderiam escolher uma alternativa que poderia resultar em um ganho menor, mas certo de, por exemplo, 240 dólares, ou poderiam escolher a alternativa que representava uma menor probabilidade de ganhar uma maior quantia (1.000 dólares com 25\% de chances). Em tal condição, os participantes escolhiam mais frequentemente a alternativa certa e com menor magnitude, de menor risco. Em outra condição, as alternativas eram verbalmente expressas aos participantes em termos de perdas. Assim, cada participante, por exemplo, escolhia entre uma perda certa de 750 dólares versus $25 \%$ de chances de não perder nada. Nesse caso, a maioria dos participantes preferia a segunda alternativa de risco. Em todas as manipulações, as recompensas (reforçadores) foram hipotéticas.

Nas pesquisas de analistas do comportamento que envolveram um modelo parecido também houve manipulações em que a variável atraso do reforço era manipulada ao invés da probabilidade do reforço. Por exemplo, um determinado participante deveria escolher entre uma alternativa que envolvia um reforçador imediato e de menor magnitude versus outra alternativa cuja escolha resultaria em um reforçador de maior magnitude, dias, semanas ou ainda anos após a escolha. Nessas pesquisas, de um modo geral, diferentes atrasos, probabilidades e magnitudes de reforçadores eram manipulados e seus efeitos eram medidos sobre as escolhas de humanos adultos. Nessa literatura, os dados encontrados sugerem que há pesquisas que argumentam que os efeitos do atraso e da probabilidade do reforço sobre a escolha em esquemas concorrentes são semelhantes (Jones \& Rachlin, 2009; Rachlin et al., 1986; Rachlin et al., 1991). Por outro lado, outras pesquisas sugerem que os efeitos são diferentes e que a manipulação de diferentes magnitudes para a opção representando a quantia de maior probabilidade ou atrasada foi crítica para isso (Christensen, Parker, Silberberg, \& Hursh, 1998; Coelho, Hanna, \& Todorov, 2003; Green, Myerson, \& Ostaszewski, 1999; Myerson, Green, Hanson, Holt, \& Estle, 2003). É importante destacar que, diferentemente do estudo de Rachlin et al. (1987), os reforçadores programados nos demais estudos envolveram reforçadores monetários hipotéticos. Levantamento sistemático foi realizado por Green e Myerson (2004) sobre comparações de atraso e probabilidades.

Na condição 2 de probabilidade do reforçador da presente pesquisa, nas fases que se seguiram àquela com FR (SigFR, FI e CRF), entretanto, para P5 e P6, os dados foram diferentes daqueles obtidos nas mesmas fases da condição $1 \mathrm{com}$ atraso do reforço. Os participantes em questão alteraram seu padrão de responder, com maior escolha de reforço menor e mais provável. Esse dado sugere que os parâmetros de atraso e probabilidade do reforço apresentam efeitos diferentes sobre as escolhas de participantes humanos adultos quando outros esquemas de reforço (que não o FR) concorrentes são manipulados. Os dados, entretanto, sugerem semelhanças com aqueles que foram produzidos por pombos (Siegel \& Rachlin, 1995) em condições com esquemas de reforço semelhantes, embora as contingências de reforço tenham sido diferentes no sentido que o estudo com os pombos envolveu a manipulação de magnitude e atraso do reforço. Seria importante uma pesquisa com pombos com um delineamento e condições semelhantes de esquemas concorrentes que avaliassem os efeitos do parâmetro da probabilidade do reforço sobre as escolhas de pombos. Esse dado favoreceria uma comparação mais precisa entre os desempenhos de pombos e humanos (dados do presente estudo) quando há manipulação de probabilidade do reforço. Essa proposta de análise poderia favorecer uma melhor comparação e compreensão acerca de semelhanças e diferenças dos efeitos de diferentes parâmetros (como atraso e probabilidade do reforço) de escolha em esquemas concorrentes em organismos de diferentes espécies.

Ainda com relação à condição 2, embora $\mathrm{P} 4$ tenha sido selecionado na fase $\mathrm{CRF} 1$, seu padrão de maior escolha do componente R1 com reforçador menor e mais provável permaneceu inalterado em todos os blocos da fase FR, não tendo ocorrido, portanto, reversão de escolha. Esse dado pode ter alguma relação com o fato de $\mathrm{P} 4$ ter passado por maior exposição à fase CRF1, embora isso não fosse necessário. Ele passou por quatro blocos de tentativas da fase em questão em virtude de terem ocorrido falhas do software de coleta de dados na verificação de cumprimento dos critérios de encerramento da fase. Embora descrições verbais sobre as escolhas não tenham sido um dado sistematicamente coletado e analisado, P4 verbalizou que optou por escolher mais a alternativa que proporcionava menos pontos, mas que tinham maior probabilidade de serem adquiridos.

\section{REFERÊNCIAS}

Coelho, C., Hanna, E. S., \& Todorov, J. C. (2003). Magnitude, atraso e probabilidade de reforço em situações hipotéticas de risco. Psicologia: Teoria e Pesquisa, 19, 269-278.

Christensen, J., Parker, S., Silberberg, A., \& Hursh, S. (1998). Trade-offs in choice between risk and delay depend on monetary amounts. Journal of the Experimental Analysis of Behavior, 69, 123-139.

Ferster, C. B., \& Skinner, B.F. (1957). Schedules of reinforcement. New York: Appleton-CenturyCrofts.

Green, L., \& Rachlin, H. (1996). Commitment using punishment. Journal of the Experimental Analysis of Behavior, 65, 593-601.

Green, L., Myerson, J., \& Ostaszewski, P. (1999). Amount of reward has opposite effects on the discounting of delayed and probabilistic outcomes. Journal of Experimental Psychology: Learning, Memory, and Cognition, 25, 418-427.

Green, L., \& Myerson, J. (2004). A discounting framework for choice with delayed and probabilistic rewards. Psychological Bulletin, 130, 769-792.

Hanna, E.S., \& Todorov, J.C. (2002). Modelos de autocontrole na análise experimental do 
comportamento: utilidade e crítica. Psicologia: Teoria e Pesquisa, 18 (3), 337-343.

Jones, B.A., \& Rachlin, H. (2009). Delay, probability, and social discounting in a public godos game. Journal of the Experimental Analysis of Behavior, 91, 61-73.

Kahneman, D., \& Tversky, A. (1984). Choices, values, and frames. American Psychologist, 39, 341-350.

Logue, A.W. (1995). Self-Control: Waiting until tomorrow for what you want today. Englewood Cliffs, NJ, US: Prentice-Hall, Inc.

Matos, D. C., Del Poço, T. P., Micheletto, N., Almeida, P. E. A., Di Rienzo, V. D., \& Panetta, P. A. B. (2014). Efeitos do atraso do reforço sobre a escolha em condições com esquemas concorrentes simples variáveis. Em N. Borges, L. Aureliano e J. L. Leonardi (orgs). Comportamento em foco. vol 4. (pp.215-230). São Paulo: Associação Brasileira de Psicologia e Medicina Comportamental ABPMC.

Rachlin, H., \& Green, L. (1972). Commitment, choice and self-control. Journal of the Experimental Analysis of Behavior, 17, 15-22.

Rachlin, H., Logue, A. W., Gibbon, J., \& Frankel, M. (1986). Cognition and behavior in studies of choice. Psychological Review, 93, 33-45.

Rachlin, R., Castrogiovanni, A., \& Cross, D. (1987). Probability and delay in commitment. Journal of the Experimental Analysis of Behavior, 48, 347353.

Rachlin, H., Raineri, A., \& Cross, D. (1991). Subjective probability and delay. Journal of the Experimental Analysis of Behavior, 55, 233-244.

Rachlin, R., \& Siegel, E. (1994). Temporal Patterning in Probabilistic Choice. Organizational Behavior and Human Decision Processes, 59, 161-176.

Rachlin, H. (1994). The Context of Pigeon and Human Choice. Em S.C. Hayes, L.J. Hayes, M. Sato, \& K. Oto (Eds.), Behavior Analysis of Language and Cognition. (pp. 301-319). Reno, NV: Context Press.

Rachlin, H. (1995). Self-control: Beyond commitment. Behavioral and Brain Sciences, 18, 109-121.

Rachlin, H. (1995). The Value of Temporal Patterns in Behavior. Current Directions in Psychological Science, 4, 188-192.

Rachlin, H. (2000). The Science of Self-Control. Cambridge, London: Harvard University Press.

Siegel, E., \& Rachlin, H. (1995). Soft commitment: Self-control achieved by response persistence. Journal of the Experimental Analysis of Behavior, 64, 117-128.
Submetido em 19/08/2013. Aceito em 02/11/2014. Publicado em 01/02/2016. 